\title{
A two-grid analysis of the combination of mixed finite elements and Vanka-type relaxation
}

\author{
J. Molenaar \\ Centre for Mathematics and Computer Science \\ P.O. Box 4079, 1009 AB Amsterdam, The Netherlands
}

\begin{abstract}
In this paper a two-grid algorithm is discussed for the mixed finite element discretization of Poisson's equation. The algorithm is based on a Vanka-type relaxation; the grid transfer operators are selected in accordance with the discretization. Local mode analysis is used to show that Vanka-type relaxation is an efficient smoother indeed. By studying the Fourier transform of the error amplification matrix we find that the canonical grid transfer operators are sufficiently accurate for grid independent convergence. However, this conclusion depends on the relaxation pattern used.
\end{abstract}

\section{INTRODUCTION}

The Mixed Finite Element (MFE) method is widely used for the discretization of second order elliptic systems. For the iterative solution of the discrete systems of equations multigrid methods are proposed. In [1] we presented a multigrid method for the MFE-discretization of the stationary semiconductor equations. This multigrid method employs a symmetric block Gauss-Seidel relaxation, as proposed by Vanka [2], which seems to be an efficient smoother. The prolongation and restriction in this multigrid algorithm are the canonical choice for the lowest order Raviart-Thomas elements that are used in the discretization. However, a priori it is not clear whether these canonical grid transfer operators are accurate enough to ensure grid independent convergence of the multigrid algorithm. Therefore we study two-grid algorithms for the model equation

$$
\begin{aligned}
\operatorname{div}(\operatorname{grad} u) & =0, \text { on } \Omega, \\
u & =0, \text { on } \Omega .
\end{aligned}
$$

For ease of notation we only treat the case $\Omega \subset \mathbb{R}$. The Poisson equation (1.1) is discretized by the MFE-method (section 2). As the discretization is not stable if a sourceterm is present in (1.1) a quadrature rule is used which lumps the equations (cf. [3]). A two-grid algorithm for the discrete system is presented in section 3. We study the convergence behavior of this two-grid algorithm by Fourier analysis. The Fourier representations of the different operators in the two-grid algorithm are derived in section 4. By local mode analysis we show that Vanka-type relaxation is an efficient smoother for the discreet system indeed (section 5). In our analysis we include the use of a relaxation parameter as well as different relaxation patterns: lexicographical and red-black. In section 6 we study the error amplification matrix of the two-grid algorithm. Surprisingly we find that the required accuracy of the grid transfer operators is not only determined by the order of the differential equations (cf. [4,5]), but also depends on the relaxation pattern used. Our conclusions are summarized in the final section.

\section{Discretization}

To discretize the second order differential equation (1.1) by the mixed finite element method, we rewrite it as a system of two first order equations, 


$$
\begin{aligned}
\sigma-\operatorname{grad} u & =0, \\
\operatorname{div} \sigma & =0, \\
u(0)=u(1) & =0 .
\end{aligned}
$$

Let $L^{2}(\Omega)$ be the Hilbert space of square integrable functions on $\Omega \subset \mathbb{R}$ with inner product

$$
(u, t)=\int_{\Omega} u t d \Omega
$$

and let $H(d i v, \Omega)$ be the Hilbert space defined by

$$
H(d i v, \Omega)=\left\{\sigma \mid \sigma \in L^{2}(\Omega), \operatorname{div} \sigma \in L^{2}(\Omega)\right\}
$$

with norm

$$
\|\boldsymbol{\sigma}\|_{H(d i v, \Omega)}^{2}=\|\boldsymbol{\sigma}\|_{L^{2}(\Omega)}^{2}+\|d i v \boldsymbol{\sigma}\|_{L^{2}(\Omega)}^{2} .
$$

By introduction of the product space $\Lambda(\Omega)=H(d i v, \Omega) \times L^{2}(\Omega)$ the weak formulation of (2.1) is: find $(\sigma, u) \in \Lambda(\Omega)$, such that

$$
\begin{aligned}
\int_{\Omega} \sigma \cdot \tau d \Omega+\int_{\Omega} u d i v \tau d \Omega & =0, \quad \forall \tau \in H(\operatorname{div}, \Omega), \\
\int_{\Omega} t \operatorname{div} \sigma d \Omega & =0, \quad \forall t \in L^{2}(\Omega) .
\end{aligned}
$$

To discretize (2.2) we decompose the domain $\Omega$ into a set $\Omega_{h}$ of $N$ uniform cells $\Omega_{h}^{i}$,

$$
\Omega_{h}^{i}=\left[\frac{(i-1)}{h}, \frac{i}{h}\right], \quad i=1, \ldots, N,
$$

of size $h=\frac{1}{N}$.

On this mesh lowest order Raviart-Thomas elements are defined, which span the subspace $\Lambda_{h}(\Omega) \subset \Lambda(\Omega)$. On each cell $\Omega_{h}^{i}$ the indicator function $e_{h}^{i} \in L^{2}(\Omega)$ is defined by

$$
e_{h}^{i}(x)= \begin{cases}1, & x \in \Omega_{h}, \\ 0, & x \notin \Omega_{h} .\end{cases}
$$

For every edge at $x=j h$ of an interval $\Omega_{h}^{i}$ a piecewise linear function $\epsilon_{h}^{j} \in H(d i v, \Omega)$ is defined by

$$
\epsilon_{h}^{i}\left(E_{h}^{k}\right)=\delta_{j k}, \quad k=0, \ldots, N,
$$

where $\delta_{j k}$ denotes the Kronecker delta. The discrete approximation $\left(\sigma_{h}, u_{h}\right)$ of solution $(\sigma, u)$ is

$$
\begin{aligned}
& \sigma_{h}=\sum_{j=0, N} \sigma \epsilon_{h}, \\
& u_{h}=\sum_{i=1, N} u_{h}^{i} e_{h}^{i} .
\end{aligned}
$$

To discretize the equation we proceed as usual and we replace $\Lambda(\Omega)$ in (2.2) by $\Lambda_{h}(\Omega)$ and use $\left(\epsilon_{h}, e_{h}^{i}\right)$ as the testfunctions. After division by $h$ for proper scaling, the resulting algebraic system for $\left(\sigma_{h}, \underline{u}_{h}\right)^{T}$, i.e. the column vector of the coefficients $\left\{\sigma h, u_{h}^{i}\right\}$ is

$$
\left[\begin{array}{cc}
A_{h} & D_{h} \\
D_{h}^{T} & 0
\end{array}\right]\left[\begin{array}{l}
\underline{\sigma}_{h} \\
\underline{u}_{h}
\end{array}\right]=\left[\begin{array}{l}
0 \\
0
\end{array}\right] .
$$

The matrix elements in this system are

$$
\left(A_{h}\right)_{j k}=\frac{1}{h} \int_{\Omega} \epsilon_{h} \epsilon_{h}^{k} d \Omega= \begin{cases}\frac{1}{6}, & ,|j-k|=1, \\ \frac{2}{3}, & , j=k, \quad j \notin\{0, N\}, \\ \frac{1}{3}, & , j=k, \quad j \in\{0, N\}, \\ 0, & , \text { otherwise, }\end{cases}
$$


and

$$
\left(D_{h}\right)_{j i}=\frac{1}{h} \int_{\Omega} e_{h}^{i} d i v \epsilon \epsilon_{h}^{j} d \Omega=\left\{\begin{aligned}
-\frac{1}{h}, & , j=i-1, \\
+\frac{1}{h}, & , j=i \\
0, & , \text { otherwise. }
\end{aligned}\right.
$$

If a sourceterm is present in (1.1) this discretization is not stable in the sense that the matrix, obtained after elimination of $\sigma_{h}$, is necessarily a $M$-matrix. Therefore we change the discretization by approximating the integral in $(2.8 \mathrm{a})$ by a repeated trapezoidal rule,

$$
\left(A_{h}\right)_{j k} \approx \frac{1}{2} \sum_{i=1, N}\left(\epsilon_{h}^{j}(i h) \epsilon_{h}^{k}(i h)+\epsilon h((i-1) h) \epsilon_{h}^{k}((i-1) h)\right) .
$$

By this quadrature $A_{h}$ is approximated by a diagonal matrix; effectively the matrix $A_{h}$ is lumped. If $\sigma_{h}$ is eliminated from the lumped system, we obtain a $M$-matrix indeed, even if a sourceterm is present.

In this paper we analyze two-grid algorithms for the solution of the linear system (2.7), both for the lumped and the non lumped case. In fact we treat the more general situation

$$
\left(A_{h}\right)_{j k}=\left\{\begin{aligned}
\kappa, & |j-k|=1, \\
1-2 \kappa, & j=k, \quad j \notin\{0, N\}, \\
\frac{1}{2}-\kappa, & j=k, \quad j \in\{0, N\}, \\
0, & \text { otherwise, }
\end{aligned}\right.
$$

which implies the lumped case $(\kappa=0)$ as well as exact integration $\left(\kappa=\frac{1}{6}\right)$.

\section{TWO-GRID ALGORITHM}

In this section we discuss the different operators in a two-grid algorithm for the iterative solution of the system of equations (2.7). The discrete fine grid operator $L_{h}: \mathbb{R}^{2 N+1} \rightarrow \mathbb{R}^{2 N+1}$ is defined by the system (2.7),

$$
L_{h}=\left(\begin{array}{cc}
A_{h} & D_{h} \\
D_{h}^{T} & 0
\end{array}\right) .
$$

The coarse grid is obtained by cellwise coarsening, i.e. by taking $H=2 h$ in (2.3). This implies that the approximating subspaces are nested, $\Lambda_{H}(\Omega) \subset \Lambda_{h}(\Omega)$; hence the canonical grid transfer operators are available. The canonical prolongation $P_{h}: \mathbb{R}^{N+1} \rightarrow \mathbb{R}^{2 N+1}$ is define on the space of coefficient vectors $\left(\sigma_{H}, u_{H}\right)^{T}$; the canonical restriction $R_{H}: \mathbb{R}^{2 N+1} \rightarrow \mathbb{R}^{N}$ is the adjoint of $P_{h}$. The coarse grid operator is obtained by using the same discretization on the coarse grid $\Omega_{H}$ as on the fine grid $\Omega_{h}$. If exact quadrature is used $\left(\kappa=\frac{1}{6}\right)$, we find that $L_{H}$ is the Galerkin approximation of $L_{h}$ :

$$
L_{H}=R_{H} L_{h} P_{h} \text {. }
$$

As smoothing operator $S_{h}: \mathbb{R}^{2 N+1} \rightarrow \mathbb{R}^{2 N+1}$ we use symmetric block Gauss-Seidel relaxation. By this method all cells are scanned in some determined order, and in each cell $\Omega_{h}^{i}$ the three equations corresponding to the testfunctions $\epsilon_{h}^{i}, \epsilon_{h}^{i-1}$ and $e_{h}^{i}$, are solved for $\sigma_{h}^{i-1}, \sigma_{h}^{i}$ and $u_{h}^{i}$. After each update a next cell is visited. In this paper we consider both lexicographical (SBGS) and red-black ordering (SBRB) ordering of the cells.

Finally we define the two-grid error amplification matrix

$$
M_{h}^{\nu_{2} \nu_{1}}=S_{h}^{\nu_{2}}\left(I_{h}-P_{h}\left(L_{H}\right)^{-1} R_{H} L_{h}\right) S_{h}^{\nu_{1}},
$$


where $I_{h}: \mathbb{R}^{2 N+1} \rightarrow \mathbb{R}^{2 N+1}$ denotes the identity operator and $v_{1}, v_{2}$ the number of pre- and post relaxation sweeps, respectively.

4. FOURIER ANALYSIS: THE COARSE GRID CORRECTION In order to derive Fourier representations of the different operators in the two-grid algorithm, we extend the domain to $\Omega=\mathbb{R}$ and omit the boundary conditions. The coefficient vectors $\sigma_{h}$ and $u_{h}$ are considered as gridfunctions defined on different discretization grids

$$
\mathbb{Z}_{h, s}=\{(j-s) h \mid j \in \mathbb{Z}\},
$$

with

$$
s= \begin{cases}0, & \text { for } \underline{\sigma}_{h}, \\ \frac{1}{2}, & \text { for } \underline{u}_{h} .\end{cases}
$$

The space of discrete $L_{2}$-functions on $\mathbb{Z}_{h, s}$, denoted by

$$
L_{h, s}\left(\mathbb{Z}_{h, s}\right)=\left\{\left.f_{h, s}\left|f_{h, s}: \mathbb{Z}_{h, s} \rightarrow \mathbb{C} ; h \sum_{j}\right| f_{h, s}((j-s) h)\right|^{2}<\infty\right\},
$$

is a Hilbert space. The Fourier transform $F T\left(f_{h, s}\right)=\hat{f}_{h, s}: T_{h} \rightarrow \mathbb{C}$ of a $L_{h, s}$-function is defined by

$$
\left.\hat{f}_{h, s}(\omega)=\frac{h}{\sqrt{2 \pi}} \sum_{j \in \mathbf{Z}} e^{-i(j-s) h \omega} f_{h, s}(j-s) h\right),
$$

with $T_{h}=\left(-\frac{\pi}{h}, \frac{\pi}{h}\right]$. The inverse transformation is given by

$$
f_{h, s}((j-s) h)=\frac{1}{\sqrt{2 \pi}} \int_{\omega \in T_{h}} e^{i(j-s) h \omega} \hat{f}_{h, s}(\omega) d \omega .
$$

By Parseval's equality the Fourier transformation operator $F T: L_{h, s} \rightarrow L_{2}\left(T_{h}\right)$ is an unitary operator.

Convolution or Toeplitz operators $B_{h}: L_{h, s} \rightarrow L_{h, s}$ are linear operators, generated by a gridfunction $b_{h, 0} \in L_{h, 0}$ :

$$
B_{h} f_{h, s}((j-s) h)=\sum_{k \in \mathbb{Z}} b_{h, 0}(k h) f_{h, s}((j-k-s) h) .
$$

The Fourier transform $F T\left(B_{h}\right)=\hat{B}_{h}$ of a Toeplitz operator $B_{h}$ is defined by

$$
\hat{B}_{h}(\omega)=\hat{b}_{h, 0}(\omega) \text {. }
$$

For example, the matrices $A_{h}$ and $D_{h}$ in (2.7) are Toeplitz operators with Fourier transforms

$$
\hat{A}_{h}(\omega)=1-4 \kappa \sin ^{2}\left(\frac{h \omega}{2}\right)
$$

and

$$
\hat{D}_{h}(\omega)=\frac{2 i}{h} \sin \left(\frac{h \omega}{2}\right)
$$

In order to obtain Fourier representations of the grid transfer operators we introduce the elementary prolongation $P_{h, s}^{0}: L_{H, s} \rightarrow L_{h, s}$,

$$
\left(P_{h, s}^{0} f_{H, s}\right)((j-s) h)= \begin{cases}f_{H, s}\left(\left(\frac{\dot{1}}{2}-s\right) h\right), & j \text { even, } \\ 0, & j \text { odd, }\end{cases}
$$

and the elementary restriction $R_{H, s}^{0}: L_{h, s} \rightarrow L_{H, s}$,

$$
\left(R_{H, s}^{0} f_{h, s}\right)((j-s) H)=f_{h, s}((2 j-s) h) .
$$


Using (4.2), (4.3) and (4.8) we find that the Fourier transforms of $f_{h, s}$ and $R_{H, s}^{0} f_{h, s}$ are related by

$$
\left(R_{H, s}^{0} f_{h, s}\right)(\omega)=e^{i s h \omega} \sum_{p=0,1} e^{-i p s \pi} \hat{f}_{h, s}\left(\omega+p \frac{\pi}{h}\right),
$$

with $\omega \in T_{H} \subset T_{h}$. Notice that $R_{H, s}^{0}$ aliases two frequencies $\left\{\omega, \omega+\frac{\pi}{h}\right\} \in T_{h}$ with one frequency $\omega \in T_{H}$. The frequencies $\omega \in T_{H}$ are called low frequencies in $T_{h}$ and the $\omega \in T_{h} / T_{H}$ are high frequencies. Now every $\omega \in T_{h}$ can be written as a 2-vector $\left(\omega+p \frac{\pi}{h}\right), p \in\{0,1\}$ on $T_{H}$. Hence for $\hat{f}_{h, s} \in L_{2}\left(T_{h}\right)$ we may also use the notation $\mathrm{f}_{h, s}$, where $\mathrm{f}_{h, s}$ is a 2-vector with entries $\hat{f}_{h, s}\left(\omega+p \frac{\pi}{h}\right)$. Consistent with this notation, we write the Fourier transform $\hat{B}_{h, s}(\omega)$ of a Toeplitz operator as a $2 \times 2$-diagonal matrix $\hat{\mathbf{B}}_{h, s}(\omega)$ with entries $\hat{B}_{h, s}\left(\omega+p \frac{\pi}{h}\right)$. Any restriction operator $R_{H, s}$, that is defined by an unique stencil, can be written as the combination of $R_{H, s}^{0}$ and a Toeplitz operator $B_{h}$. Hence the Fourier transform of $R_{H, s}$ is given by

$$
\hat{\mathbf{R}}_{H, s}(\omega)=\hat{\mathbf{R}}_{H, s}^{0}(\omega) \hat{\mathbf{B}}_{h}(\omega)
$$

where $\hat{\mathbf{R}}_{H, s}(\omega)$ and $\hat{\mathbf{R}}_{H, s}^{0}(\omega)$ are $1 \times 2$ matrices.

Analogous to the restriction we may write any prolongation $P_{h, s}$ as the combination of $P_{h, s}^{0}$ and a Toeplitz operator $B_{h}$. The Fourier transforms of $f_{h, s}$ and $P_{h, s}^{0} f_{H, s}$ are related by

$$
\left(P_{h, s}^{0} f_{H, s}\right)\left(\omega+p \frac{\pi}{h}\right)=\frac{1}{2} e^{-i s h\left(\omega-p \frac{\pi}{h}\right)} \hat{f}_{H, s}(\omega)
$$

The Fourier transform of $P_{h, s}=B_{h} P_{h, s}^{0}$ is in matrix notation

$$
\hat{\mathbf{P}}_{h, s}(\omega)=\hat{\mathbf{B}}_{h}(\omega) \hat{\mathbf{P}}_{h, s}^{0}(\omega),
$$

where $\hat{\mathbf{P}}_{h, s}$ and $\hat{\mathbf{P}}_{h, s}^{0}$ are $2 \times 1$-matrices. Using (4.11) and (4.12) we compute the Fourier transform of the canonical prolongation $P_{h}$ (see section 3) as

$$
\hat{\mathbf{P}}_{h}(\omega)=\left[\begin{array}{cc}
\hat{\mathbf{P}}_{h}^{\sigma}(\omega) & 0 \\
0 & \hat{\mathbf{P}}_{h}^{u}(\omega)
\end{array}\right]=\left[\begin{array}{cc}
\cos ^{2} \frac{\theta}{2} & 0 \\
\sin ^{2} \frac{\theta}{2} & 0 \\
0 & \cos \frac{\theta}{2} \\
0 & \sin \frac{\theta}{2}
\end{array}\right],
$$

with $\theta=h \omega$. The Fourier transform $\hat{\mathbf{R}}_{H}(\omega)$ of the canonical restriction $R_{H}$ is the transposed of $\hat{\mathbf{P}}_{h}(\omega)$.

The components of the Fourier transform of a grid transfer operator are trigoniometric functions of $\theta$; this allows us to classify them according to their behavior in the case $\omega$ fixed and $h \rightarrow 0$. Suppose that the Fourier transforms of a prolongation and its adjoint restriction are given by $\hat{\boldsymbol{\Pi}}_{h, s}(\omega)$ and $\hat{\Pi}_{h, s}^{T}(\omega)$, respectively. The low frequency order $m_{L}$ of a grid transfer operator $\Pi_{h, s}$ is the largest number $m_{L} \geqslant 0$ such that

$$
\hat{\Pi}_{h, s}(\omega)=1+O\left(\theta^{m_{l}}\right), \quad \text { for } h \rightarrow 0, \omega \in T_{H} .
$$

The high frequency order $m_{H}$ of $\Pi_{h, s}$ is the largest number $m_{H} \geqslant 0$ for which

$$
\hat{\Pi}\left(\omega+\frac{\pi}{h}\right)=O\left(\theta^{m_{H}}\right), \text { for } h \rightarrow 0, \omega \in T_{H} \text {. }
$$

For the canonical grid transfer operators we have: $m_{L}^{o}=2, m_{H}^{\sigma}=2, m_{L}^{u}=2$ and $m_{H}^{u}=1$. To avoid large amplification of high frequencies, the high frequency order $m_{H}$ should be at least equal to the order of the differential equation (cf. [4,5]). As we are considering a system of first order equations, 
we conclude that the canonical grid transfer operators are sufficiently accurate. In fact, the Fourier transform of the coarse grid correction operator $M_{h}^{00}$ is given by

$$
\hat{\mathbf{M}}_{h}^{00}(\omega)=\left(\begin{array}{cccc}
+\sin ^{2} \frac{\theta}{2} & -\cos ^{2} \frac{\theta}{2} & 0 & 0 \\
-\sin ^{2} \frac{\theta}{2} & +\cos ^{2} \frac{\theta}{2} & 0 & 0 \\
\frac{h}{2 i \sin \frac{\theta}{2}} \gamma_{L}\left(\frac{\theta}{2}\right) & \frac{h}{2 i \sin \frac{\theta}{2}} \gamma_{H}\left(\frac{\theta}{2}\right) & \sin ^{2} \frac{\theta}{2} & -\frac{1}{2} \sin \theta \\
\frac{h}{2 i \cos \frac{\theta}{2}} \gamma_{L}\left(\frac{\theta}{2}\right) & \frac{h}{2 i \cos \frac{\theta}{2}} \gamma_{H}\left(\frac{\theta}{2}\right) & -\frac{1}{2} \sin \theta & \cos ^{2} \frac{\theta}{2}
\end{array}\right),
$$

with $\gamma_{L}(\theta)=\sin ^{2} \theta\left(1-12 \kappa \cos ^{2} \theta\right)$ and $\gamma_{H}(\theta)=\cos ^{2} \theta\left(1-12 \kappa \sin ^{2} \theta\right)$.

So, if it is assumed that $\inf _{\omega \in T_{H}}|\omega|$ is bounded away from zero by the boundary conditions, we see that all elements of $\mathbf{M}_{h}^{00}(\omega)$ remain bounded for $h \rightarrow 0$. This implies that errors in $\left(\sigma_{h}, u_{h}\right)$ are not blown up by the coarse grid correction if $h \rightarrow 0$.

\section{FOURIER ANALYSIS: RELAXATION}

In this section we derive the Fourier representation of the smoothing operators SBGS and SBRB. We start by treating the lexicographical ordering of the cells. In a single SBGS-sweep the $\sigma_{h}$ are updated twice; so starting from initial values $\left\{\sigma_{h}^{j}, u_{h}^{i}\right\}$ SBGS yields new values $\left\{\bar{\sigma}_{h}^{j}, \bar{u}_{h}^{i}\right\}$, using intermediate values $\tilde{\sigma}_{h}^{j}$. If the cells are visited from left to right, we have in every cell $\Omega_{h}^{i}$

$$
\begin{aligned}
\frac{1}{h}\left(\bar{\sigma}_{h}^{i}-\bar{\sigma}_{h}^{i-1}\right) & =0, \\
\kappa \sigma_{h}^{i+1}+(1-2 \kappa) \tilde{\sigma}_{h}^{i}+\kappa \bar{\sigma}_{h}^{i-1}+\frac{1}{h}\left(u_{h}^{i+1}-\bar{u}_{h}^{i}\right) & =0, \\
\kappa \tilde{\sigma}_{h}^{i}+(1-2 \kappa) \bar{\sigma}_{h}^{i-1}+\kappa \bar{\sigma}_{h}^{i-2}+\frac{1}{h}\left(\vec{u}_{h}^{i}-\bar{u}_{h}^{i-1}\right) & =0 .
\end{aligned}
$$

Starting with a Fourier mode $\sigma h=a e^{i j h \omega}$ and $u_{h}^{j}=b e^{i\left(j-\frac{1}{2}\right) h \omega}$ we see that $\tilde{\sigma}_{h}^{j}=\tilde{a} e^{i j h \omega}, \bar{\sigma}_{h}^{j}=\bar{a} e^{i j h \omega}$ and $\bar{u}_{h}^{j}=\bar{b} e^{i\left(j-\frac{1}{2}\right) h \omega}$. After elimination of $\tilde{a}$ from (5.1) we obtain a relation between the components before and after relaxation,

$$
\left(\begin{array}{l}
\bar{a} \\
b
\end{array}\right]=\hat{\mathbf{S}}_{h}^{G S}(\omega)\left(\begin{array}{l}
a \\
b
\end{array}\right)
$$

with

$$
\hat{\mathbf{S}}_{h}^{G S}(\omega)=\frac{e^{i \frac{\theta}{2}}}{2-2 \kappa-(1-2 \kappa) e^{-i \theta}}\left[\begin{array}{ll}
\kappa e^{i \frac{\theta}{2}}\left(1-e^{i \theta}\right) & \frac{1-e^{i \theta}}{h} \\
\kappa h\left(\kappa+(1-\kappa) e^{i \theta}\right) & e^{-i \frac{\theta}{2}}\left(\kappa+(1-\kappa) e^{i \theta}\right)
\end{array}\right] .
$$

The spectral radius $\rho(\cdot)$ and the spectral norm $\|\cdot\|_{s}$ of $\hat{\mathbf{S}}_{h}^{G S}(\omega)$ are respectively

$$
\rho\left(\hat{\mathbf{S}}_{h}^{G S}(\omega)\right)=\left|\frac{1-2 i \kappa \sin \theta}{2-2 \kappa-(1-2 \kappa) e^{-i \theta}}\right|
$$

and 


$$
\left\|\hat{\mathbf{S}}_{h}^{G S}(\omega)\right\|_{S}=\left(1+\kappa^{2} h^{2}\right) \frac{\left[\frac{4}{h^{2}} \sin ^{2} \frac{\theta}{2}+1-4 \kappa \sin \frac{\theta}{2}+4 \kappa^{2} \sin ^{2} \frac{\theta}{2}\right]^{\frac{1}{2}}}{\left|2-2 \kappa-(1-2 \kappa) e^{-i \theta)}\right|} .
$$

By using (5.4a), the smoothing factor $\mu^{C S}=\sup \rho\left(\hat{\mathbf{S}}_{h}^{G S}(\omega)\right)$ is readily calculated: $\frac{\pi}{2} \leqslant|\theta| \leqslant \pi$

$$
\mu^{G S}= \begin{cases}\left(\frac{1}{5}\right)^{\frac{1}{2}}, & \kappa=0, \\ \left(\frac{10}{29}\right)^{\frac{1}{2}}, & \kappa=\frac{1}{6},\end{cases}
$$

independent of $h$.

From (5.4b) we see that $\left\|\hat{\mathbf{S}}_{h}^{G S}(\omega)\right\|_{S}$ becomes unbounded for $h \rightarrow 0$. This is a consequence of the fact that, starting from an initial error $\sigma_{h}=0$, we find errors in $\bar{\sigma}_{h}$ of magnitude $O\left(h^{-1}\right.$ ) for $h \rightarrow 0$ (cf. 5.3). From the boundedness of $\rho\left(\hat{\mathbf{S}}_{h}^{G S}(\bar{\omega})\right)$ we conclude that only in the first relaxation sweep errors in $\sigma_{h}$ are blown up by SBGS. In order to obtain a measure of what happens in the first sweep, we introduce the scaled norm $\|\cdot\|_{H}$, which is defined by

$$
\left\|\hat{\mathbf{A}}_{h}(\omega)\right\|_{H}=\left\|\hat{\mathbf{H}}_{h} \hat{\mathbf{A}}_{h}(\omega)\right\|_{S},
$$

with $H_{h}: \mathbb{R}^{2 N+1} \rightarrow \mathbb{R}^{2 N+1}$ a scaling operator,

$$
H_{h}\left[\begin{array}{l}
\sigma_{h} \\
\underline{u}_{h}
\end{array}\right]=\left[\begin{array}{l}
h \sigma_{h} \\
\underline{u_{h}}
\end{array}\right] .
$$

With respect to this norm we find for SBGS:

$$
\left\|\hat{\mathbf{S}}_{h}^{G S}(\omega)\right\|_{H}=\left(1+\kappa^{2} h^{2}\right) \frac{\left(4 \sin ^{2} \frac{\theta}{2}+1-4 \kappa \sin \frac{\theta}{2}+4 \kappa^{2} \sin ^{2} \frac{\theta}{2}\right)^{\frac{1}{2}}}{\left|2-2 \kappa-(1-2 \kappa) e^{-i \theta}\right|},
$$

which is bounded for $h \rightarrow 0$, indeed.

Vanka proposes underrelaxation for $\sigma_{h}$ (cf. [2]) to improve the smoothing properties of SBGS. This can be analyzed by replacing $\tilde{\sigma}_{h}^{j}$ an $\bar{\sigma}_{h}^{j-1}$ in $(5.1)$ by $\frac{1}{\alpha}\left(\tilde{\sigma}_{h}^{j}-(1-\alpha) \sigma_{h}^{j}\right)$ and $\frac{1}{\alpha}\left(\sigma_{h}^{j-1}-(1-\alpha) \tilde{\sigma}_{h}^{j-1}\right)$, respectively, where $\alpha$ denotes the relaxation parameter. For $k=0$ the smoothing factor of this damped relaxation is easily derived and it is (independent of $h$ ) given by

$$
\mu^{G S}(\alpha)=\max \left(\left(\frac{1}{5}\right)^{\frac{1}{2}},(1-\alpha)^{2}\right) .
$$

Fig. 5.1 shows a graph of $\mu^{G S}(\alpha)$ in the case $k=\frac{1}{6}$ (no lumping). Numerically we find an optimal smoothing rate $\mu^{C S}\left(\alpha_{o p t}\right)=0.369$ for $\alpha_{\text {opt }}=0.4583$.

The Fourier representation of SBRB relaxation is obtained by a similar method. As usual we write $S_{h}^{R B}$ as the product of the partial step operators $S_{h}^{R}$ and $S_{h}^{B}$,

$$
\begin{aligned}
& \hat{\mathbf{S}}_{h}^{R B}(\omega)=\hat{\mathbf{S}}_{h}^{R}(\omega) \hat{\mathbf{S}}_{R}^{B}(\omega) \\
& \hat{\mathbf{S}}_{h}^{R}(\omega)=\left[\begin{array}{rrrr}
s_{1}(\theta) & s_{2}(\theta+\pi) & s_{3}(\theta) & s_{4}(\theta+\pi) \\
s_{2}(\theta) & s_{1}(\theta+\pi) & s_{4}(\theta) & s_{3}(\theta+\pi) \\
s_{5}(\theta) & -i s_{5}(\theta+\pi) & s_{7}(\theta) & -i s_{8}(\theta+\pi) \\
i s_{5}(\theta) & s_{5}(\theta+\pi) & i s_{8}(\theta) & s_{7}(\theta+\pi)
\end{array}\right], \\
& {\left[\hat{\mathbf{S}}_{h}^{B}(\omega)\right]_{i j}=(-1)^{i+j}\left[\hat{\mathbf{S}}_{h}^{R}(\omega)\right]_{i j},}
\end{aligned}
$$


with

$$
\begin{array}{ll}
s_{1}(\theta)=\kappa f(\theta) e^{i \frac{3 \theta}{2}} \cos \frac{\theta}{2}, & s_{2}(\theta)=-i \kappa f(\theta) e^{i \frac{3 \theta}{2}} \sin \frac{\theta}{2}, \\
s_{3}(\theta)=2 i \frac{f(\theta)}{h} \sin \theta \cos \frac{\theta}{2}, & s_{4}(\theta)=2 \frac{f(\theta)}{h} \sin \theta \sin \frac{\theta}{2}, \\
s_{7}(\theta)=e^{i \frac{\theta}{2}} \cos \frac{\theta}{2}+i(1-\kappa) f(\theta) \sin \theta, & s_{8}(\theta)=i e^{i \frac{\theta}{2}} \sin \frac{\theta}{2}+i(1-\kappa) f(\theta) \sin \theta, \\
s_{5}(\theta)=\frac{h \kappa}{2} e^{i \frac{3 \theta}{2}}(1+(1-\kappa) f(\theta)), &
\end{array}
$$

and

$$
f(\theta)=\frac{-1}{2-2 \kappa+\kappa e^{-2 i \theta}} .
$$

We see that all elements of $\hat{\mathbf{S}}_{h}^{R B}(\omega)$ remain bounded for $h \rightarrow 0$ and $\omega$ fixed. However by doing so, we only consider the limit cases $|\theta| \rightarrow 0$, and $|\theta| \rightarrow \pi$; the spectral norm of $\hat{\mathbf{S}}_{h}^{R B}(\omega)$ becomes unbounded for $h \rightarrow 0$ and $\theta$ fixed. Numerical computation shows that the scaled norm of $\hat{\mathbf{S}}_{h}{ }^{R B}(\omega)$ is bounded:

$$
\sup _{0 \leqslant|h \omega| \leqslant \pi}\left\|\mathbf{S}_{h}^{R B}(\omega)\right\|_{H}<\infty, \text { for } h \rightarrow 0 .
$$

As SBRB relaxation mixes low and high frequencies the smoothing factor $\mu^{R B}$ is defined by

$$
\mu^{R B}=\sup _{\frac{\pi}{2}<|h \omega|<\pi} \rho\left(\hat{\mathbf{Q}} \hat{\mathbf{S}}_{h}^{R B}(\omega)\right),
$$

where $\hat{\mathbf{Q}}$ denotes the operator that annihilates all low frequencies

$$
\hat{\mathbf{Q}}=\left[\begin{array}{llll}
0 & & & \\
& 1 & & \\
& & 0 & \\
& & & 1
\end{array}\right] .
$$

If underrelaxation of $\sigma h$ is taken into account, we obtain for $\kappa=0$

$$
\mu^{R B}(\alpha)=\max \left(\frac{1}{8},(1-\alpha)^{2}\right),
$$

independent of $h$. A plot of $\mu^{R B}(\alpha)$ for $\kappa=\frac{1}{6}$ is shown in Fig 5.2. In this case underrelaxation hardly improves the smoothing factor; numerically' we find $\mu^{R B}(\alpha=1)=0.127$.

6. FOURIER ANALYSIS: THE TWO-GRID ALGORITHM

In the previous sections we have shown that appropriate norms of the coarse grid correction operator and the relaxation operator remain bounded in the limit case of vanishing meshsize. However, this does not imply that the scaled norm of the two-grid error amplification matrix is bounded, and hence that the convergence rate of the two-grid algorithm is mesh-independent. If $\kappa=0$ both SBGS and SBRB relaxation without damping $(\alpha=1)$ eliminate $\sigma_{h}$, so we are solving a second order differential equation for $u_{h}$. Therefore we may expect that the canonical grid transfer operators $P_{h}^{u}$ and $R_{H}^{u}$ are not accurate enough.

We show that this is the case indeed by studying the two-grid algorithm with SBGS relaxation for $\kappa=0$. If a single SBGS-sweep is used for pre- and post smoothing, the Fourier transform of $M_{h}^{11}$ after $n$ cycles is given by

$$
\left(\hat{\mathbf{M}}_{h}^{11}(\omega)\right)^{n}=\left(\frac{e^{2 i \theta}}{4-e^{-2 i \theta}}\right)^{n}\left[\begin{array}{ll}
0 & M^{\sigma u} \\
0 & M^{u u}
\end{array}\right)
$$




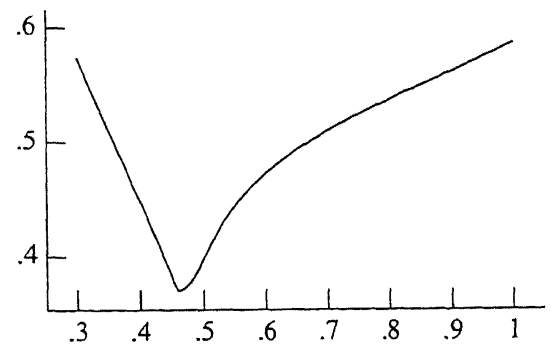

Fig. 5.1. Smoothing factor $\mu^{G S}$ depending on the relaxation parameter $\alpha$

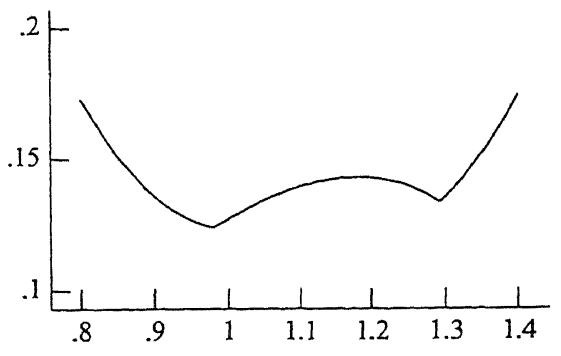

Fig. 5.2. Smoothing factor $\mu^{R B}$ depending on the relaxation parameter $\alpha$

$$
\begin{array}{llcc} 
& & \left(P_{h}, R_{H}\right) & \left(\bar{P}_{h}, \tilde{R}_{H}\right) \\
\cline { 3 - 4 } \kappa=0 & \text { SBGS } & \infty & 0.346 \\
& \text { SBRB } & 0.590 & 0.099 \\
\kappa=1 / 6 & \text { SBGS }(\alpha=1) & \infty & 0.531 \\
& \text { SBGS }\left(\alpha=\alpha_{\text {opt }}\right) & \infty & 0.368 \\
& \text { SBRB } & 0.628 & 0.339
\end{array}
$$

TABLE 6.1. Scaled norm of the two-grid error amplification matrix, sup $\left\|\hat{\mathbf{M}}_{h}^{11}(\omega)\right\|_{H}$. $0 \leqslant|\theta| \leqslant \frac{\pi}{2}$

\begin{tabular}{lll|ll} 
& \multicolumn{2}{c|}{$\left(P_{h}, R_{H}\right)$} & \multicolumn{2}{c}{$\left(\tilde{P}_{h}, \tilde{R}_{H}\right)$} \\
\cline { 2 - 5 }$v$ & SBGS & SBRB & SBGS & SBRB \\
& & & & \\
1 & 0.577 & 0.500 & 0.343 & 0.096 \\
2 & 0.333 & 0.325 & 0.145 & 0.046 \\
3 & 0.192 & 0.259 & 0.088 & 0.031 \\
4 & 0.111 & 0.221 & 0.058 & 0.023
\end{tabular}

TABLE 6.2. Two-level convergence factor $\lambda_{\nu}, \kappa=0$.

\begin{tabular}{lccc|ccc} 
& \multicolumn{3}{c|}{$\left(P_{h}, R_{H}\right)$} & \multicolumn{3}{|c}{$\left(\tilde{P}_{h}, \tilde{R}_{H}\right)$} \\
\cline { 2 - 7 }$v$ & $\begin{array}{c}\text { SBGS } \\
(\alpha=1)\end{array}$ & SBGS & SBRB & SBGS & SBGS & SBRB \\
$\left(\alpha=\alpha_{\text {opt }}\right)$ & & & & & \\
$\left(\alpha=\alpha_{\text {opt }}\right)$ & \\
1 & 0.657 & 0.477 & 0.457 & 0.476 & 0.391 & 0.264 \\
2 & 0.429 & 0.211 & 0.367 & 0.311 & 0.277 & 0.178 \\
3 & 0.281 & 0.147 & 0.314 & 0.236 & 0.212 & 0.136 \\
4 & 0.184 & 0.093 & 0.278 & 0.177 & 0.166 & 0.111
\end{tabular}

TABLE 6.3. Two-level convergence factor $\lambda_{\nu}, \kappa=\frac{1}{6}$. 
with

$$
\begin{aligned}
& n \text { even : } M^{\sigma u}=\left(\begin{array}{cc}
-\frac{2}{i h} \sin \frac{\theta}{2} & 0 \\
0 & \frac{2}{i h} \cos \frac{\theta}{2}
\end{array}\right], \quad M^{u u}=\left[\begin{array}{cc}
1 & 0 \\
0 & 1
\end{array}\right], \\
& n \text { odd : } M^{\sigma u}=\left[\begin{array}{cc}
0 & \frac{2}{i h} \cos \frac{\theta}{2} \\
\frac{2}{i h} \sin \frac{\theta}{2} & 0
\end{array}\right], \quad M^{i u}=\left[\begin{array}{cc}
0 & \cot \frac{\theta}{2} \\
\tan \frac{\theta}{2} & 0
\end{array}\right] .
\end{aligned}
$$

The two-grid algorithm exhibits a typical alternating convergence behavior. An initial high frequency mode $\underline{u}_{h}$ of amplitude $b$, causes a low frequency mode of amplitude $b \cot \frac{\theta}{2}$ after a single two-grid cycle; so if $h \rightarrow 0$ initial high frequency error modes in $u_{h}$ are blown up. In the next cycle the large low frequency mode $u_{h}$ is nicely removed by the coarse grid correction, although a small high frequency error mode is introduced. This alternating behavior is reflected by the scaled norm of $\left(\mathbf{M}_{h}(\omega)\right)^{n} ;$ for $h \rightarrow 0$ we find

$$
\sup _{0 \leqslant|h \omega|<\frac{\pi}{2}}\left\|\left(\hat{\mathbf{M}}_{h}^{11}(\omega)\right)^{n}\right\|_{H}=\left\{\begin{aligned}
\left(\frac{5}{3^{n}}\right)^{\frac{1}{2}}, & n \text { even, } \\
\infty, & n \text { odd. }
\end{aligned}\right.
$$

By numerical computation we observe a similar alternating convergence behavior for $\kappa=\frac{1}{6}$, even though the coarse grid operator $L_{H}$ satisfies Galerkin's relation.

The obvious cure is to use more accurate grid transfer operators. We introduce $\tilde{P}_{h}^{u}$ the linear interpolation operator for $u_{h}$ and $\widetilde{R}_{H}^{u}$ its adjoint. The Fourier transforms of these more accurate grid transfer operators are

$$
\hat{\overrightarrow{\mathbf{P}}}_{h}(\omega)=\left[\begin{array}{cc}
\hat{\mathbf{P}}_{h}^{\sigma}(\omega) & 0 \\
0 & \hat{\tilde{\mathbf{P}}}_{h}^{u}(\omega)
\end{array}\right]=\left[\begin{array}{cc}
\cos ^{2} \frac{\theta}{2} & 0 \\
\sin ^{2} \frac{\theta}{2} & 0 \\
0 & \cos ^{3} \frac{\theta}{2} \\
0 & \sin ^{3} \frac{\theta}{2}
\end{array}\right]
$$

and

$$
\hat{\tilde{\mathbf{R}}}_{H}(\omega)=\left[\begin{array}{cc}
\hat{\mathbf{R}}_{H}^{\sigma}(\omega) & 0 \\
0 & \hat{\tilde{\mathbf{R}}}_{H}{ }_{H}(\omega)
\end{array}\right]=\left[\hat{\tilde{\mathbf{P}}}_{h}(\omega)\right]^{T},
$$

so we have $m_{L}^{u}=2$ and $m_{H}^{u}=3$. Although it is not necessary to use $\tilde{P}_{h}^{u}$ for keeping the scaled norm of the error amplification matrix bounded, it is introduced to avoid similar problems, with the

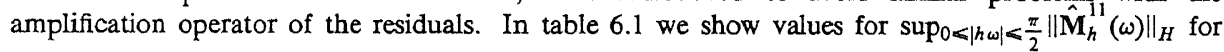
the different possible two-grid algorithms. If SBRB relaxation is used, the canonical grid transfer operators $\left(m_{h}^{\mu}=1\right)$ are sufficient: the high frequencies are so efficiently smoothed that they don't cause any problems. Here we see that the choice of the grid transfer operators is not only determined by 
the order of the differential equations, but is also influenced by the relaxation scheme.

The scaled norm of $\hat{\mathbf{M}}_{h}^{11}(\omega)$ only indicates what happens in a single two-grid cycle; the convergence rate after many cycles is estimated by the two-level convergence factor

$$
\lambda_{v}=\sup _{0 \varangle|\theta| \leqslant \frac{\pi}{2}} \rho\left(\hat{\mathbf{M}}_{h}^{v_{1} \nu_{2}}\right)
$$

with $v=v_{1}+v_{2}$. In table 6.2 we show $\lambda_{v}$ for $\kappa=0$ and for different values of $v$. The combination of the transfer operators $\tilde{P}_{h}$ and $\tilde{R}_{h}$, and SBRB relaxation leads to a fast converging algorithm. In table 6.3 we show $\lambda_{\nu}$ for $\kappa=\frac{1}{6}$. We see that the introduction of a damping parameter $\alpha$ in SBGS relaxation indeed leads to faster convergence, but the best convergence factors are again obtained by using the combination of SBRB relaxation and the transfer operators $\tilde{P}_{h}$ and $\tilde{R}_{H}$.

\section{ConClusions}

By local mode analysis we have shown that symmetric block Gauss-Seidel relaxation is an efficient smoother indeed. Although lumping of the discrete equations spoils the Galerkin property of the coarse grid operator it generally leads to faster converging two-grid algorithms. The Fourier transform of the two-grid error amplification operator shows that the canonical grid transfer operators are insufficiently accurate in the $1 D$ case if a lexicographical ordering of the gridpoints is used in the relaxation procedure; however they suffice if a red-black ordering is used.

\section{REFERENCES}

[1] MolenaAR, J., and P.W. HemKer (1990). A multigrid approach for the solution of the $2 D$ semiconductor equations, IMPACT, to appear.

[2] VANKA, S.P. (1986). Block-Implicit Multigrid Solution of Navier-Stokes Equations in Primitive Variables, J. Comput. Phys., 65, 138-158.

[3] POLAK, S.J. W.H.A. SCHILDERS and H.D. Couperus (1988). A finite element method with current conservation, in Simulation of Semiconductor Devices and Processes, Vol. 3, Bologna, Technoprint.

[4] BrandT, A. (1982). Guide to multigrid development, in Multigrid Methods, Springer Verlag, Lecture Notes in Mathematics 960.

[5] HEMKER, P.W. (1990). On the order of prolongations and restrictions in multigrid procedures, J. Appl. Math., to appear. 